\title{
Design of Delta-Sigma $\Sigma \Delta$ based Fractional N PLL Frequency Synthesizer for GSM Mobile Systems
}

\author{
Saad Wasmi Osman Luhaib \\ Assistant Lecturer \\ Dr. Khalid Khaleel Mohammed \\ Assistant Professor
}

Electrical Engineering- Mosul University, Iraq

\begin{abstract}
This paper presents the design of Delta-Sigma $\Sigma \Delta$ based fractional N PLL frequency synthesizer for GSM mobile systems. The loop filter is one of main element in fractional-N $\Sigma \Delta$ synthesizer because it shapes the noise spectrum of quantization error and reduce the spurious level in the pass band of PLL. The effect of loop filter order is studied and it is shown that by increasing the order of the loop filter, the phase noise performance will be improved, although this requires careful design consideration, as the PLL is prone instability. It's shown that the $3^{\text {rd }}$ order loop filter and $3^{\text {rd }}$ order $\Sigma \Delta$ modulator gives the best performance with reducing phase noise $(13 \mathrm{dBc} / \mathrm{Hz})$ and spurious noise $(44 \mathrm{dBc})$ of the output signal.
\end{abstract}

Keyword: PLL, Frequency Synthesizer, $\Sigma \Delta$ Modulator, Fractional-N, GSM mobile systems.

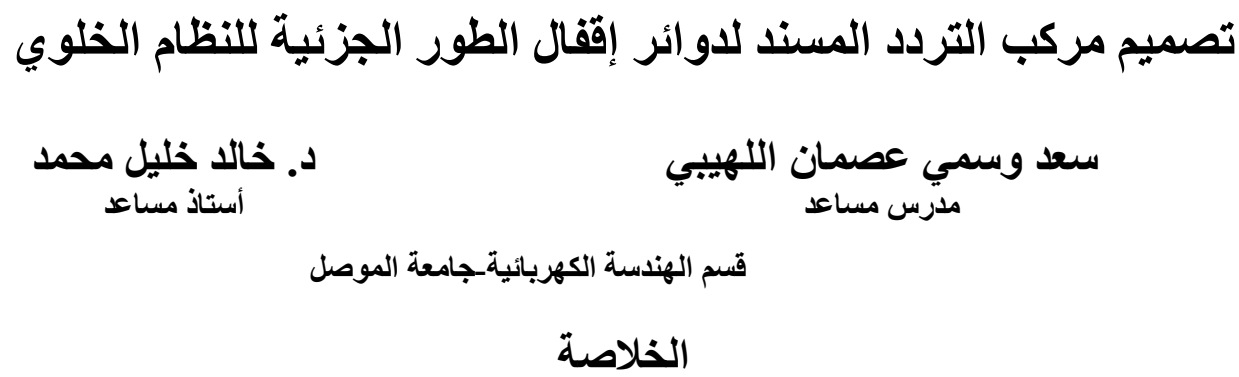

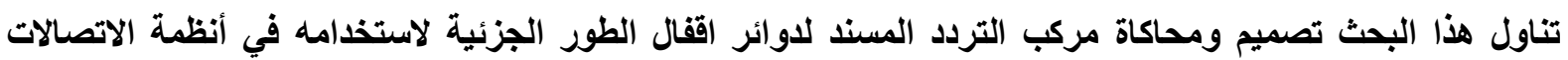

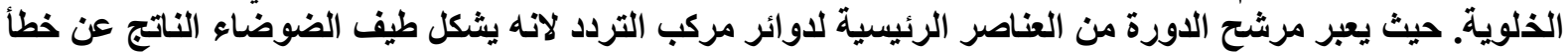

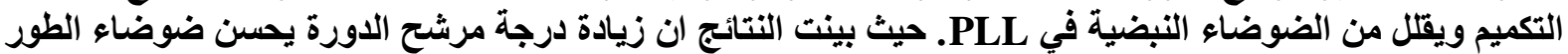

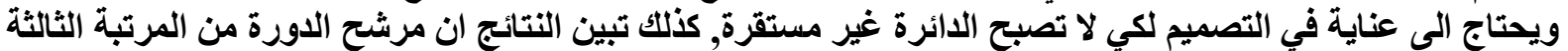

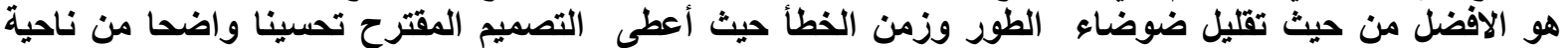

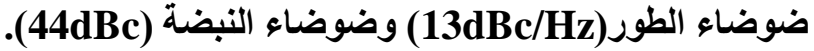




\section{Introduction}

Oscillators and frequency synthesizers are key elements in a radio system that provide a controlled frequency source for received signal down conversion and for transmitted signal up conversion. A stand-alone oscillator or a voltage controlled oscillator (VCO) does not have the required frequency stability to satisfy the low phase noise requirement. Therefore frequency synthesis is necessary to obtain accurate high frequencies from a precise low frequency crystal oscillator [1].

Scott E. Meninger designed the fractional N synthesizer with $1^{\text {st }}$ order loop filter and $1^{\text {st }} \Sigma \Delta$ modulator at $3.6 \mathrm{GHz}$ [2]. The Charlotte $\mathrm{Y}$. Lau designed the fractional $\mathrm{N}$ synthesizer using direct closed loop realization algorithm at third order $\Sigma \Delta$ [3]. In this paper using PLL Design Assistance Program (PDA) to designed the loop filter with $2^{\text {nd }}$ and $3^{\text {rd }}$ order and the $\Sigma \Delta$ modulator is chosen to be of the $1^{\text {st }}$ to $5^{\text {th }}$ order in order to select a best performance.

Frequency mixing utilizes a mixer to multiply two signals together, generating a signal that contains both the sum and the difference of the two input frequencies as shown in Fig(1). Typically, only one of these two frequencies is required. This type of synthesizer has very good noise performance and the same stability as the reference oscillator. Frequency mixing is accomplished by using a nonlinear element such as a diode or a transistor.

Unfortunately, the non-linearity's tend to produce a signal that contains many spurious tones at various mixing products and the final signal will be a combination of many frequencies with the from $n f_{a} \pm m f_{b}$ where the $f_{a}$ and $f_{b}$ are, respectively input frequency and mixed frequency and $n \& m$ are integer number. These spurious frequencies are the basic disadvantage of frequency mixing [4].

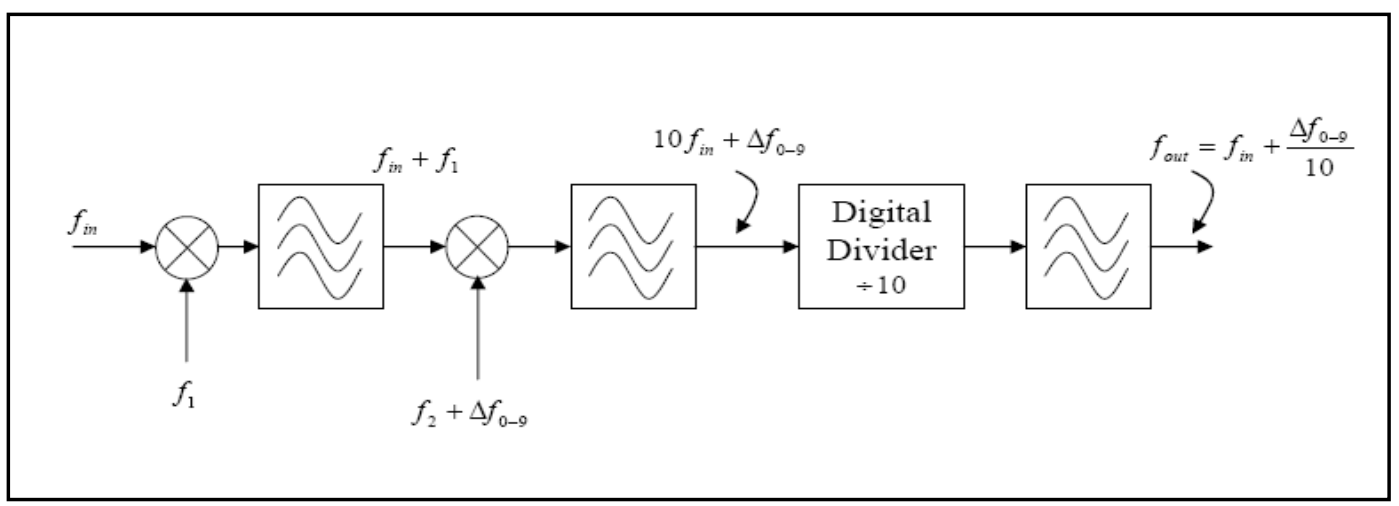

Fig. 1 Frequency synthesis using frequency mixing method

Phase locked loops (PLL) are often used to provide negative feedback in frequency synthesizers to suppress the phase noise due to oscillators.

The fractional-N technique modifies the classical indirect phase-locked loops to effectively permit the feedback divider to take a periodically changing division ratio and hence to give a non-integer ratio. For instance, if a fractional value of $1 / 8$ is required, then the division ratio changes once (from $\mathrm{N}$ to $\mathrm{N}+1$ ) every eighth cycle. 
The architecture of a fractional-N synthesizer is shown in $\operatorname{Fig}(2)$, the accumulator is a convenient device to control the division ratio because the number of occurrences of its carryout equals the numerator of the fraction [4].

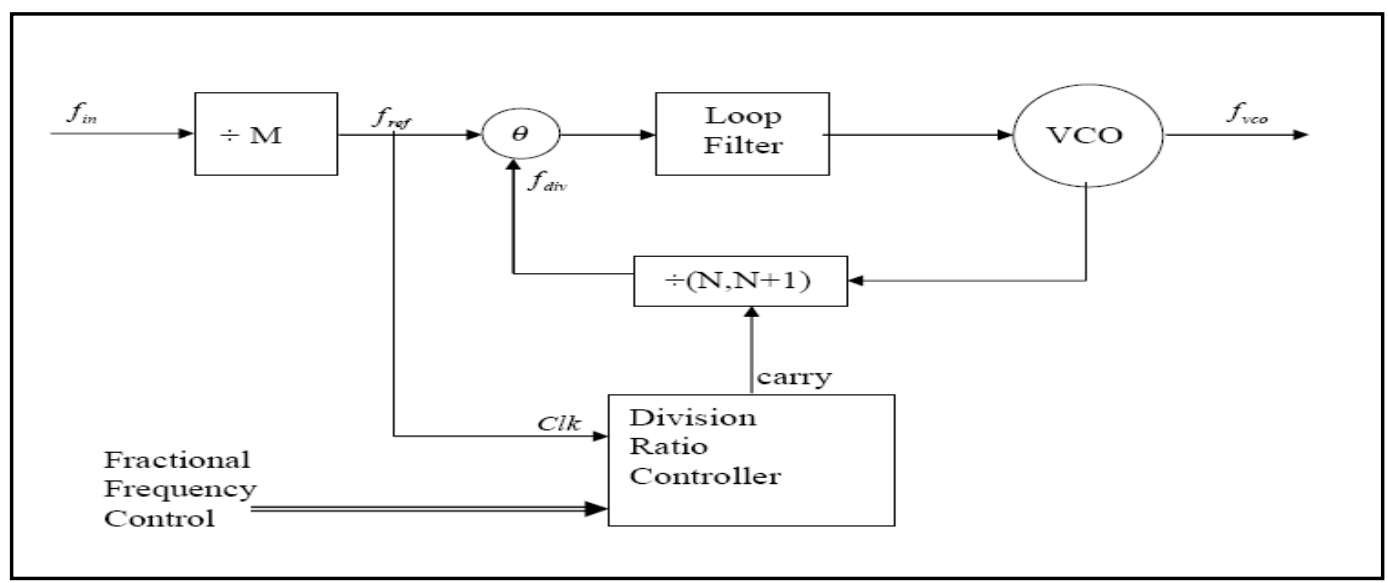

Fig. 2 Conventional fractional-N svnthesizer

The manipulation of the division ratio in Fig(2) generates phase perturbations, and hence spurious signals, which have to be eliminated in the synthesizer. The nature of the phase perturbations is predictable and can be canceled out using an analogue correction system. If the $\mathrm{VCO}$ output frequency is now changed from $N . f_{\text {ref }}$ to $(N . g) \cdot f_{\text {ref }}$ while maintaining the feedback division ratio as $\mathrm{N}$, the phase detector phase error takes on a sawtooth shape in $\operatorname{Fig}(3)[2,4,5]$ is given by

$$
\begin{aligned}
& \frac{\Delta \theta}{\Delta \mathrm{T}}=\left(\frac{N+0 . g}{N}\right) f_{r e f} \cdot T\left(\frac{2 \pi}{T}\right)-f_{r e f} T\left(\frac{2 \pi}{T}\right) \\
& \frac{\Delta \theta}{\Delta \mathrm{T}}=\left(\frac{2 \pi f_{r e f} 0 . g}{N}\right) \bmod 2 \pi
\end{aligned}
$$

where $0 . \mathrm{g}$ denotes the fractional portion of the division ratio, $\mathrm{T}$ is the time of one period.

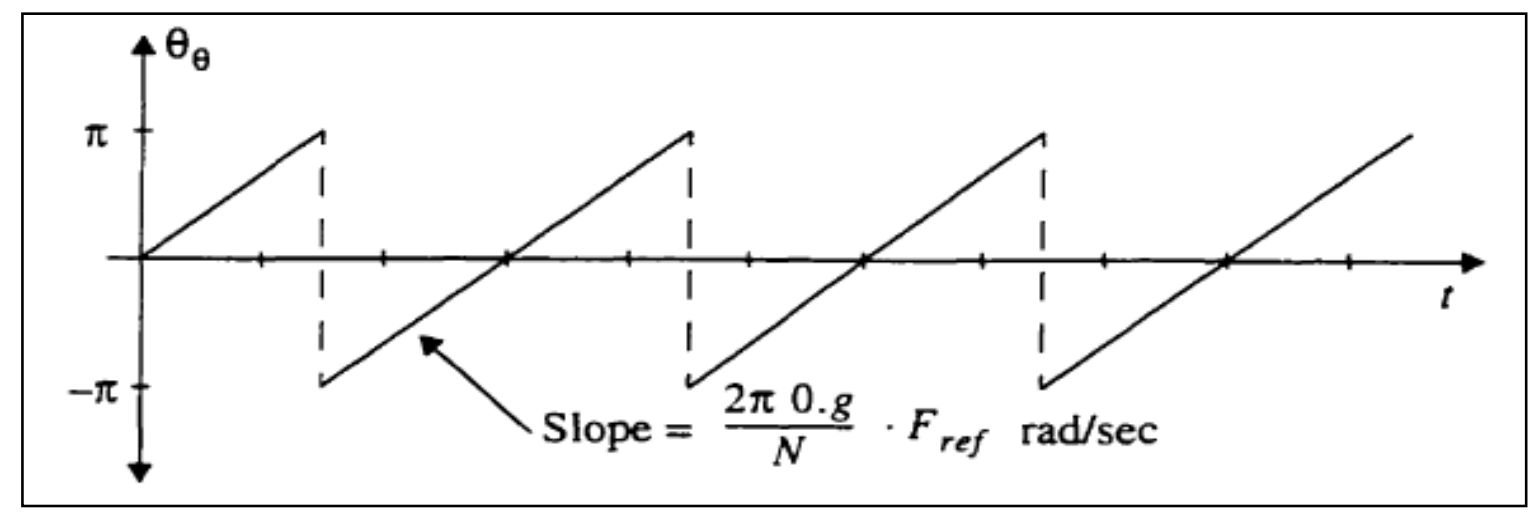

Fig. 3 Beat note from the output of the phase detector 


\section{The proposed System}

Fig(4) shows fractional-N synthesizer with high speed phase selection $[2,3,6]$. It is proposed that the loop filter is to be designed with $2^{\text {nd }}$ and $3^{\text {rd }}$ order in order to examined the synthesizer for best performance, also the $\Sigma \Delta$ modulator is to be examined with the order variation from $1^{\text {st }}$ to the $5^{\text {th }}$. The overall system with variable loop filter order and variable $\Sigma \Delta$ modulator is tested in order to reduce the phase noise, spurious noise and the r.m.s jitter time.

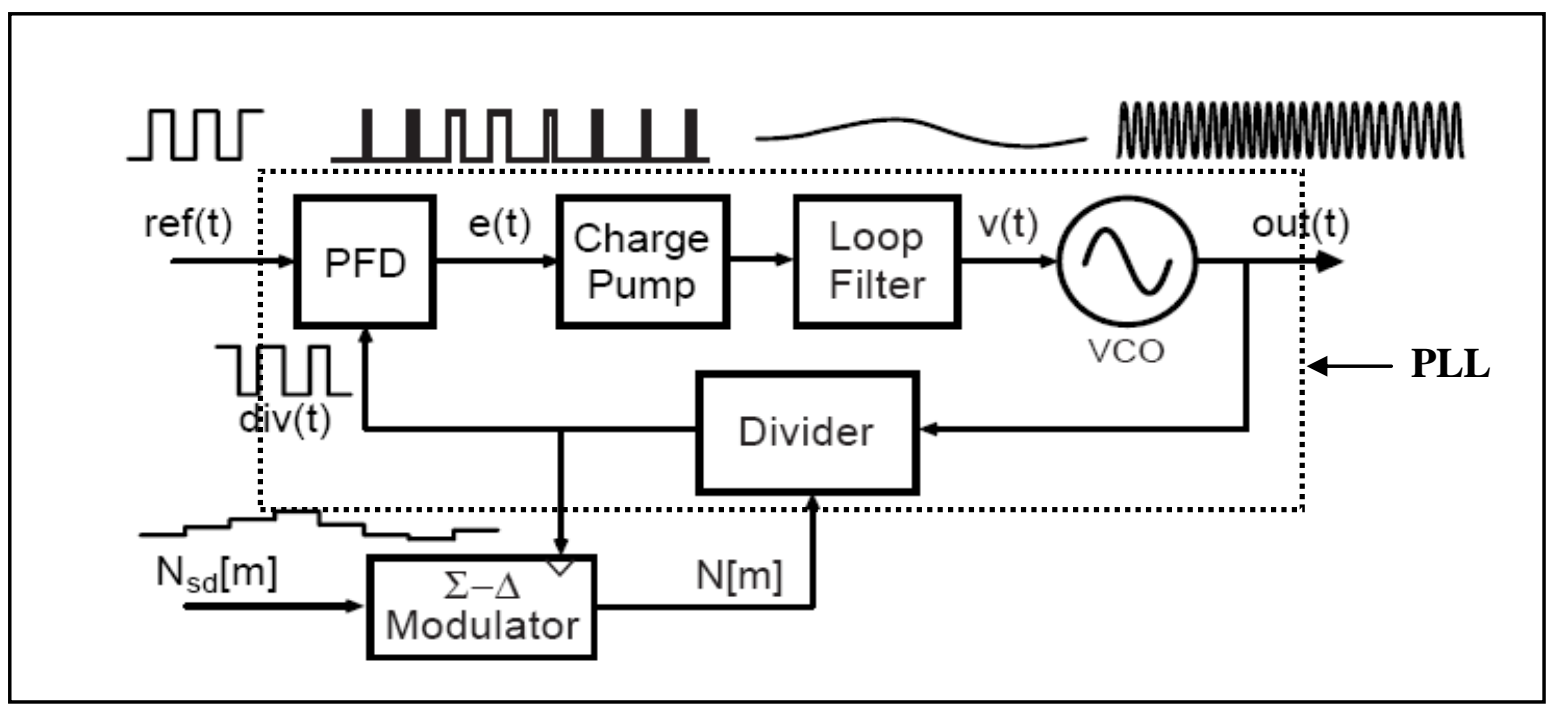

Fig. 4 The proposed $\Sigma \Delta$ fractional-N synthesizer

As shown in Fig(4),the PLL still acts as a digitally-controlled frequency generator, the phase modulator produces a bit stream, $\mathrm{N}[\mathrm{m}]$, that contains the desired frequency control embedded in a stream of binary number. The average density of binary numbers controls the division ratio of the PLL and hence the output frequency of the synthesizer [5]. The phase modulator will tend to shape the noise spectrum of the quantization error and reduce spurious level in the PLL output signal $[1,4]$.

\section{Simulation Result}

A Fractional-N PLL frequency synthesizer for GSM900 receiver applications with a frequency range of $880 \mathrm{MHz}$ to $915 \mathrm{MHz}$, a channel spacing of $1 \mathrm{MHz}$, and a settling time of less $(10 \mu \mathrm{sec})$ is targeted, where the reference frequency $\left(\mathrm{f}_{\mathrm{ref}}\right)$ is $25 \mathrm{MHz}[3,6,8]$.

Typically, a higher-order loop filter is used in Fractional-N PLL frequency synthesis applications to provide adequate suppression of the reference spurious, the high frequency phase noise from the $\Sigma \Delta$ modulator, can be eliminated by using high order loop filter.

The charge pump loop filter topology is a third-order passive network as shown in Fig(5). The use of a higher-order loop filter, however, requires careful design consideration, i.e., passive component must be determined theoretically. Therefore, its precision values must be implement in hardware, as the PLL is prone to instability. The average current-to-voltage transfer function of the loop filter is $[6,8]$. 


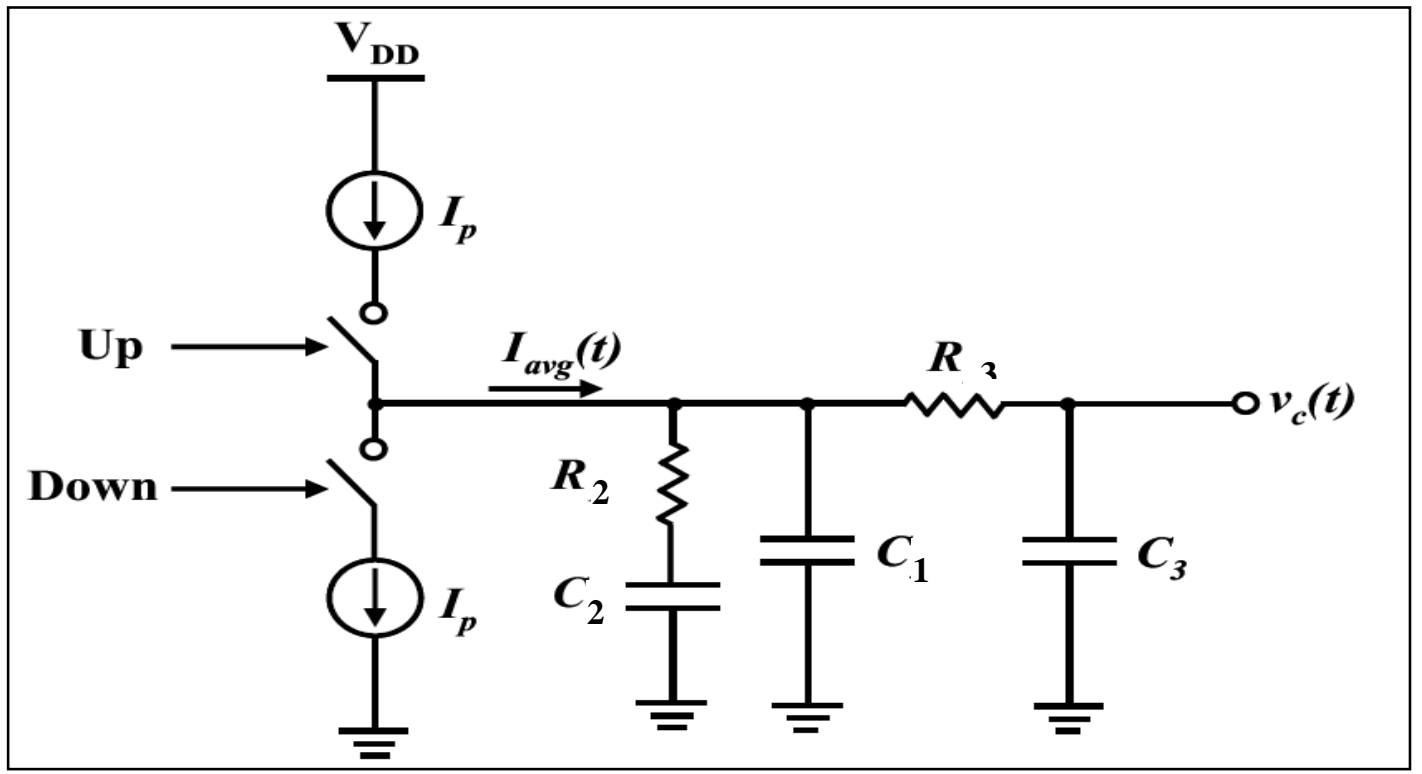

Fig. 5 A passive third-order charge pump loop filter

$$
\begin{aligned}
& H(s)=\frac{\left(1+s \tau_{2}\right)}{A_{0} \quad s\left(1+s \tau_{1}\right)\left(1+s \tau_{3}\right)} \\
& H(s)=\frac{1+s \tau_{2}}{s\left(A_{2} s^{2}+A_{1} s+A_{o}\right)} \\
& A_{\mathrm{o}}=\frac{K v \cdot I_{c p} . \alpha}{K N_{\text {norm }}}
\end{aligned}
$$

where

Nnom : Nominal divide value,

$\mathrm{Kv}$ : VCO gain, (units are assumed to be $\mathrm{Hz} / \mathrm{V}$ ),

$\alpha$ : PFD topology,

Icp : Charge pump current,. (units assumed to be Amps)

$\mathrm{K}$ : PFD constant, ,( units assumed to be Amps/rad)

$\tau_{2}=\mathrm{R}_{2} \mathrm{C}_{2}$

$\mathrm{A}_{0}=\mathrm{C}_{1}+\mathrm{C}_{2}+\mathrm{C}_{3}$

$\mathrm{A}_{1}=\mathrm{A}_{\mathrm{o}}\left(\tau_{1}+\tau_{3}\right)=\mathrm{C}_{2} \mathrm{C}_{3} \mathrm{R}_{2}+\mathrm{C}_{1} \mathrm{C}_{2} \mathrm{R}_{2}+\mathrm{C}_{1} \mathrm{C}_{3} \mathrm{R}_{3}+\mathrm{C}_{2} \mathrm{C}_{3} \mathrm{R}_{3}$

$\mathrm{A}_{2}=\mathrm{A}_{\mathrm{o}}\left(\tau_{1} \tau_{3}\right)=\mathrm{C}_{1} \mathrm{C}_{2} \mathrm{R}_{2} \mathrm{C}_{3} \mathrm{R}_{3}$

The open loop transfer function of the PLL can be determined from the following expression $[6,9]$.

$$
A(s)=\frac{K_{v} I_{c p} \alpha H(s)}{s N_{n o r m}}
$$

Where $\mathrm{N}_{\text {norm }}$ is the geometric mean of the maximum and minimum division ratio required to span the desired frequency band (in this case,36).

$$
N_{\text {norm }}=\frac{f_{\text {out }}}{f_{\text {ref }}}
$$


Where

$f_{\text {out }}$ : output frequency equal to $900 \mathrm{MHz}$.

Using PLL Design Assistance Program (PDA) and Matlab 7.4 the calculated coefficients of the $3^{\text {rd }}$ order loop filter are

$\mathrm{C}_{1}=4.1952 \mathrm{nf}, \mathrm{C}_{2}=0.34474 \mathrm{nf}, \mathrm{C}_{3}=0.52669 \mathrm{nf}, \mathrm{R}_{2}=379.373 \mathrm{ohm}, \mathrm{R}_{3}=197.3867 \mathrm{ohm} \mathrm{I}_{\mathrm{cp}}=2 \mathrm{~mA}$, $\mathrm{K}=2.193 \times 10^{12} \mathrm{Amp} / \mathrm{rad}$ and $\mathrm{K}_{\mathrm{v}}=200 \mathrm{MHz} /$ volt.

With these passive component values, the open loop transfer function of the PLL is displayed in Fig (6), where the unity gain frequency is $8.97 \mathrm{MHz}$ and the phase margin is $\left(48.4^{\circ}\right)$. This value of phase margin $\left(48.4^{\circ}\right)$ can be expressed as optimum value (more than $45^{\circ}$ and less than $90^{\circ}$ ) that gives the best stability for the system [8].

Using the parameters which are calculated in the previous section, the fractional-N

PLL frequency synthesizer is simulated using Sue2 program [7].

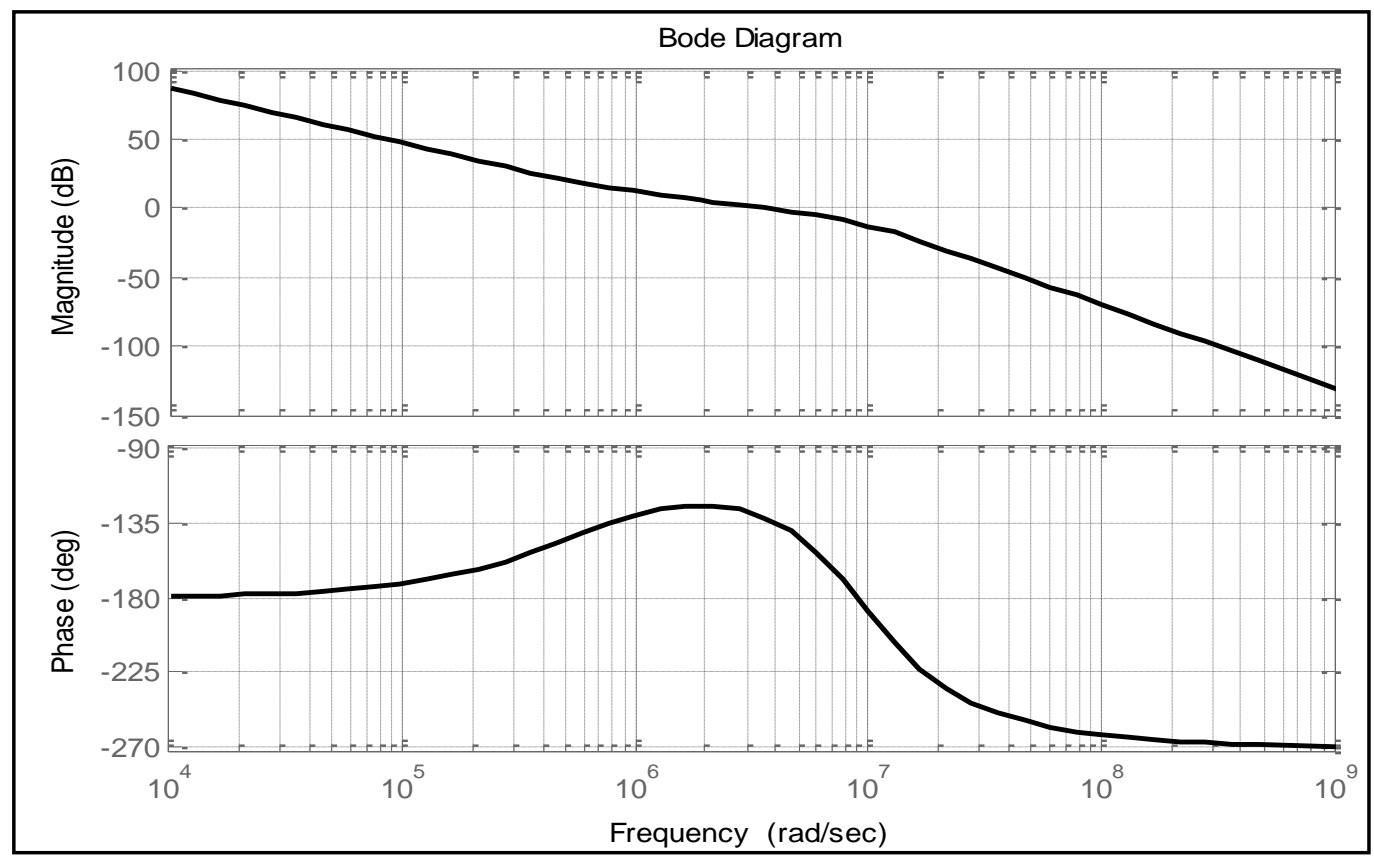

Fig. 6 Open loop transfer function of the PLL

The relationship between $\Sigma \Delta$ modulator and r.m.s jitter output is shown in $\operatorname{Fig}(7)$. It is clear that the $3^{\text {rd }}$ order give less jitter noise compared with $1^{\text {st }}$ and $2^{\text {nd }}$ order, the small deference between $4^{\text {th }}, 5^{\text {th }}$ is equal ( 0.9 pico sec).

The $\Sigma \Delta$ modulator at 3rd order multi-stage-noise-shaping (MASH) is designed according to the following equation [7].

$$
N T F=\frac{Y(z)}{R(z)}=\left(1-3 z^{-1}+3 z^{-2}-z^{-3}\right)
$$

Where NTF is noise transfer function.

The step response of the closed the loop PLL system that used in the fractional-N $\Sigma \Delta$ frequency synthesizer is shown in Fig(8),it is clear that the settling time is very small and is equal to $(5 \mu \mathrm{sec})$ 


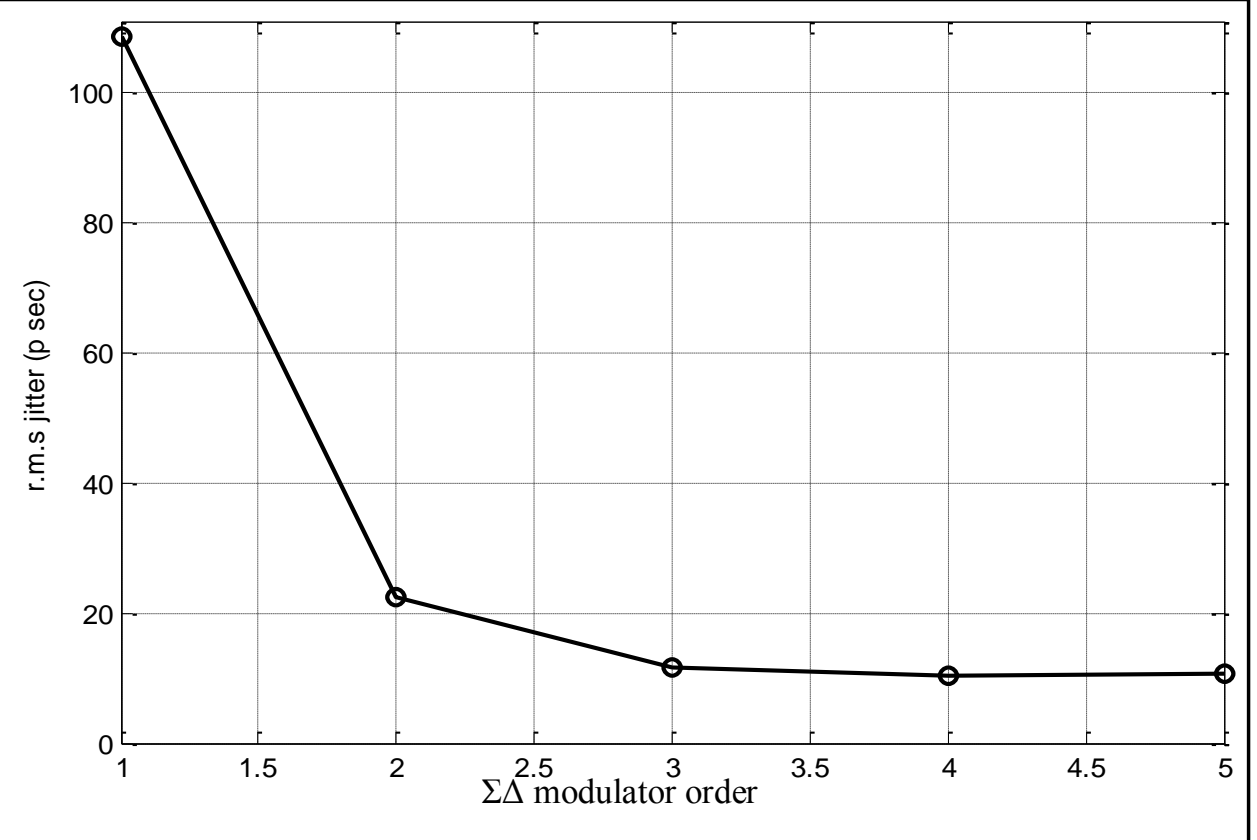

Fig. 7 Relationship between r.m.s jitter with order of $\Sigma \Delta$ modulator MASH

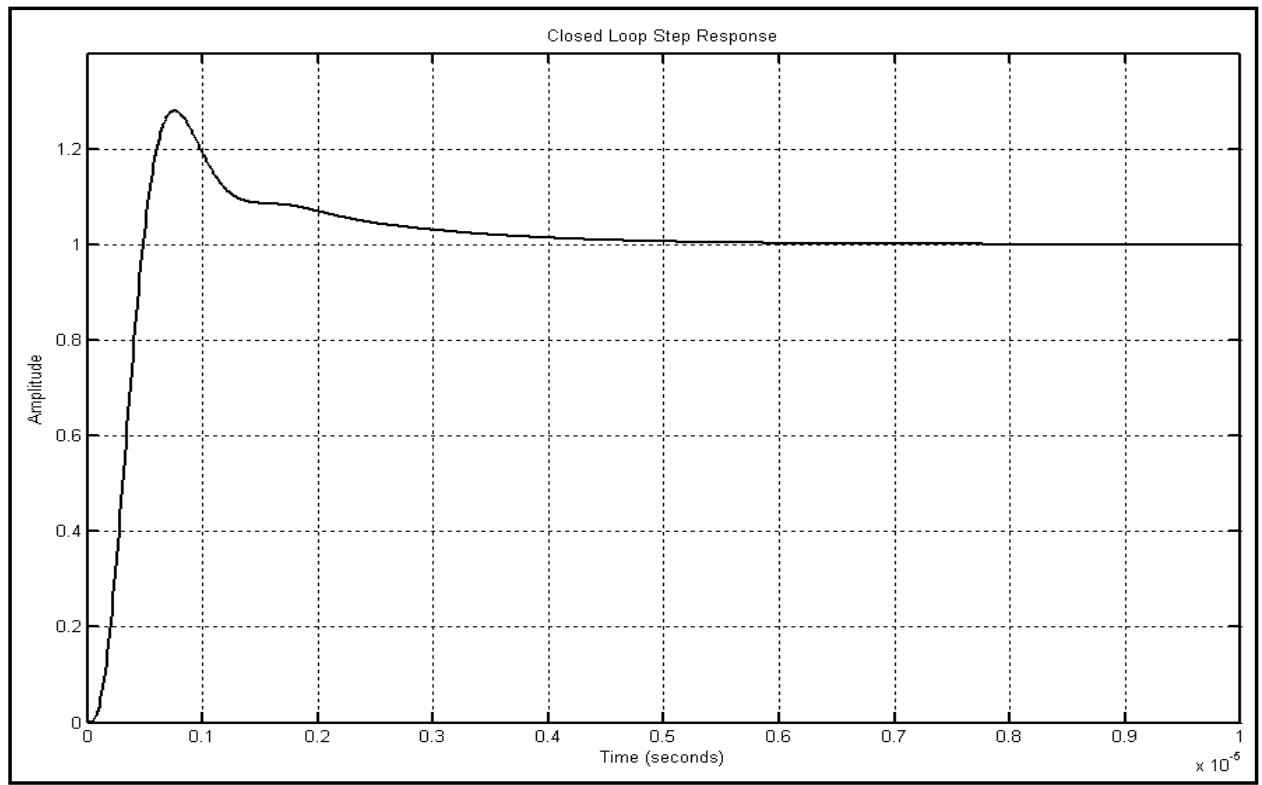

Fig. 8 Step response of PLL $3^{\text {rd }}$ order

The GSM phase noise values (L(f)) as a function of frequency offset is shown

In Fig (9) and Fig(10). The phase noise is equal to $(-140 \mathrm{dBc} / \mathrm{Hz})$ and the spurious noise is equal to $(50 \mathrm{dBc})$ at frequency offset $20 \mathrm{MHz}$ using $3^{\text {rd }}$ order $\Sigma \Delta$ modulator MASH and $2^{\text {nd }}$ order loop filter as shown in Fig(9). While, as shown in Fig(10), the phase noise is equal to $-153 \mathrm{dBc} / \mathrm{Hz})$ and the spurious noise is equal to $(6 \mathrm{dBc})$ at frequency offset $20 \mathrm{MHz}$ using $3^{\text {rd }}$ order $\Sigma \Delta$ modulator and $3^{\text {rd }}$ order loop filter.

As shown in Fig(10) it can be seen that use of $3^{\text {rd }}$ order loop filter improves the phase noise of about $(13 \mathrm{dBc} / \mathrm{Hz})$ and also improves the spurious noise of about $(44 \mathrm{dBc})$. The comparison of the proposed system with prior works is shown in Table (1). 
In the present work, the value of phase noise is smaller than the value in references [2] and [3]. Also the spurious noise is reduced by amount of $4 \mathrm{dBc}$ compared to reference [2]. The settling time and r.m.s jitter time are also reduced to value of $5 \mu$ s and $11 \mathrm{ps}$, respectively.

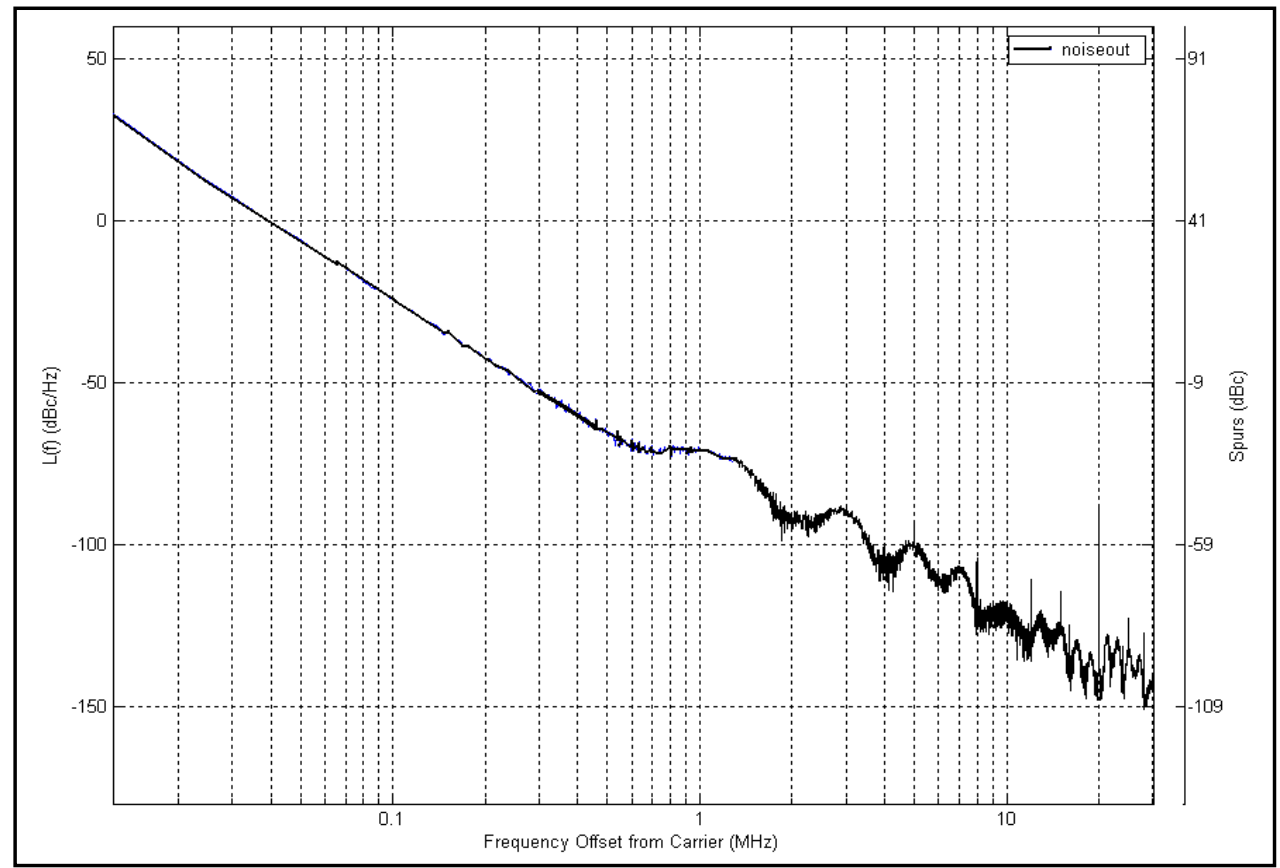

Fig. 9 Phase noise level with frequency offset for $3^{\text {rd }}$ order $\Sigma \Delta$ modulator MASH

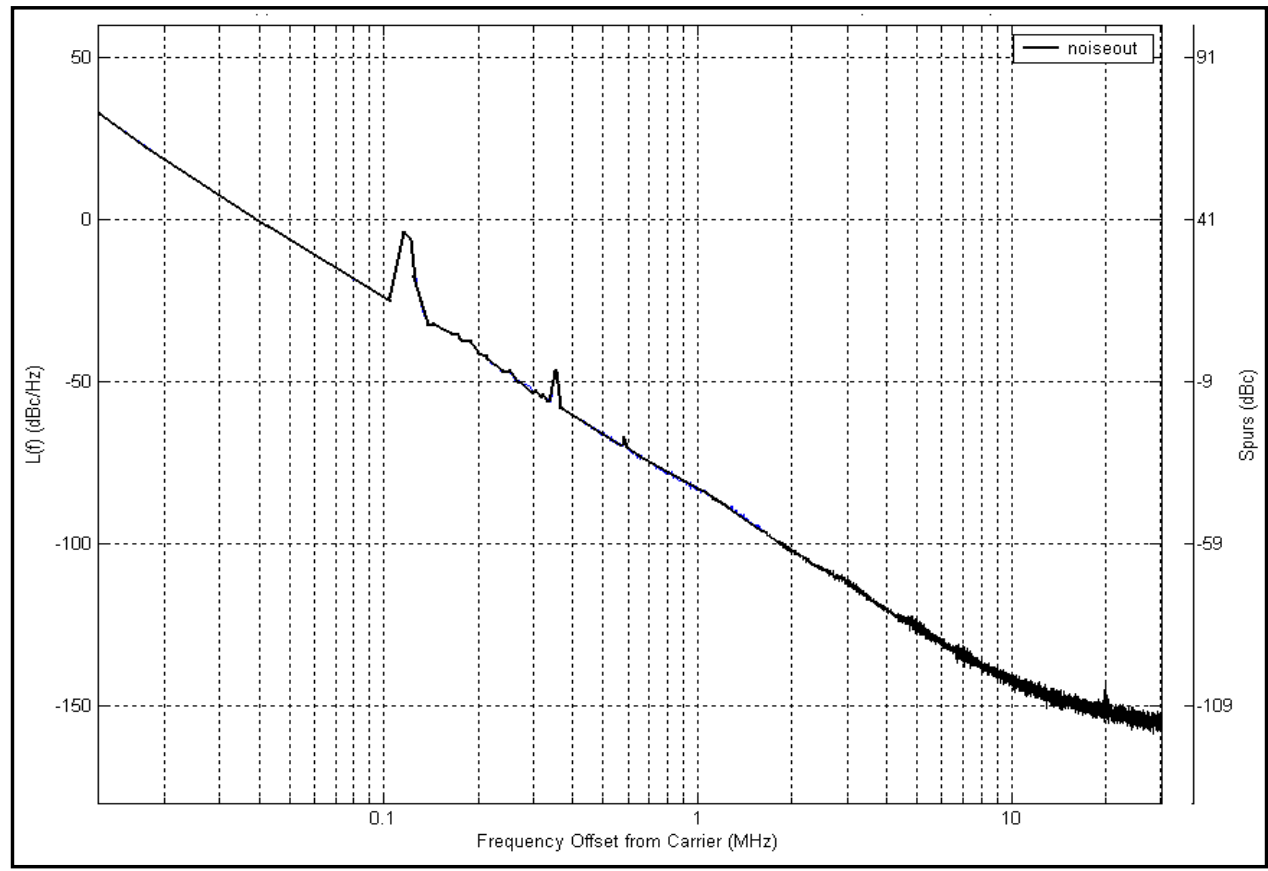

Fig. 10 Phase noise level with frequency offset for $3^{\text {rd }}$ order $\Sigma \Delta$ modulator MASH 
Table (1): Comparison with prior works.

\begin{tabular}{|l|l|l|l|}
\hline The parameter & \multicolumn{1}{|c|}{$[2]$} & \multicolumn{1}{c|}{$[3]$} & Present work \\
\hline Bandwidth & $1 \mathrm{MHZ}$ & $300 \mathrm{KHz}$ & $1 \mathrm{MHz}$ \\
\hline Loop filter order & $1^{\text {st }}$ order & $3^{\text {rd }}$ order & $2^{\text {nd }}$ and $3^{\text {rd }}$ order \\
\hline$\Sigma \Delta$ modulator order & $1^{\text {st }}$ order MASH & $3^{\text {rd }}$ order & $1^{\text {st }}-5^{\text {th }}$ order MASH \\
\hline $\begin{array}{l}\text { phase noise at frequency } \\
\text { offset } 10 \mathrm{MHz}\end{array}$ & $-141 \mathrm{dBc} / \mathrm{Hz}$ & $-143 \mathrm{dBc} / \mathrm{Hz}$ & $-145 \mathrm{dBc} / \mathrm{Hz}$ \\
\hline $\begin{array}{l}\text { Spurious noise at frequency } \\
\text { offset } 20 \mathrm{MHz}\end{array}$ & $10 \mathrm{dBc}$ & not calculated & $6 \mathrm{dBc}$ \\
\hline Jitter time & not calculated & $14.055 \mathrm{ps}$ & $11 \mathrm{ps}$ \\
\hline Settling time for PLL & not calculated & $10 \mu \mathrm{s}$ & $5 \mu \mathrm{s}$ \\
\hline
\end{tabular}

\section{Conclusion}

The fractional-N $\Sigma \Delta$ modulator frequency synthesizer has been simulated using Matlab ,PDA and Sue2 programs. The result has shows that increasing the order of the $\Sigma \Delta$ modulator from $1^{\text {st }}$ to $5^{\text {th }}$ order will improve the spurious noise performance, and the best order is the $3^{\text {rd }}$ one because it gives minimum r.m.s jitter. While increasing the order of loop filter will improve the phase noise performance and reduce the spurious noise. The open loop transfer function and the close loop transfer function prove that the system is stable because the phase margin is greater than 45 and less than 90.In this paper a considerable improvement was achieved in minimizing spurious noise level, r.m.s jitter time and settling time compared with published papers in this domain.

\section{References}

[1] R. Desikachari, "High-Speed CMOS Dual-Modulus Prescalers for Frequency Synthesis", Msc. thesis, Oregon State University, 2004.

[2] S. E. Meniger and M. H. Perrott, "Bandwidth Extension Of Low Noise Fractional-N Synthesizer", MARCO Focus Center for Circuit \& System Solutions (C2S2,www.c2s2.org), under contract 2003-CT-888.

[3] C. Y. Lau and M. H. Perrott, "Fractional-N Frequency Synthesizer Design at The Transfer Function Level Using A Direct Closed Loop Realization Algorithm", DAC 2003,Anaheim,California,USA.

[4] W. K.Tong, "A GHz CMOS Frequency Synthesizer for Mobile Communication”, Msc. thesis, University of Toronto, Department of Electrical and Computer Engineering, 1997.

[5] S.E.Meniger and M.H..Perrott, " A Dual Band 1.8GHz/900MHz, 750kb/s GMSK Transmitter Utilizing a Hybrid PFD/DAC Structure for Reduced Broadband Phase Noise”, MARCO Focus Center for Circuit \& System Solutions under contract 2003-CT888.

[6] M.H.Perrott, "PLL Design Using The PLL Design Assistance Program”,April 2005. http://www-mtl.mit.edu/ perrot.

[7] M. H. Perrott, “ CppSim (Version 3) Primer”,April 2008. http://www-mtl.mit.edu/ perrott

[8] M.Kozak and E.G.Friedman, "Design And Simulation Of Fractional -N PLL Frequency Synthesizers",IEEE ISCAS 2004,IV-780.

[9] D. Banerjee, "PLL Performance, Simulation, and Design",2006, Fourth Edition.

The work was carried out at the college of Engineering. University of Mosul 